\title{
LE MOUVEMENT 20 FEVRIER AU MAROC ENTRE L'AUTOLEGITIMATION ET LA DELEGITIMATION DE L'ÉTAT: UNE ANALYSE CRITIQUE DU DISCOURS
}

\author{
Adil Moustaoui Srhir
}

Universidad Complutense de Madrid

http://dx.doi.org/10.5209/rev_NOMA.2013.v39.n3.48322

Les mobilisations, les protestations sociales et les révoltes que connaissent les pays du Moyen-Orient et d'Afrique du Nord, dans le cadre du Printemps Arabe, sont certes le résultat d'un certain nombre de transformations culturelles et démographiques (Béchir Ayari et Geisser, 2011). Mais ils sont surtout à lire dans le contexte de ces dynamiques de changement politique, sociale et économique, qui coïncident au fond avec n'importe quel mouvement de protestation qui prétend à des mutations politiques et sociales sur le pouvoir ou au niveau des modèles de gouvernance en franchissant une structure traditionnelle de l'autoritarisme de l'État. Nous pensons que la principale raison de ces manifestations est la crise de légitimité institutionnelle, politique, sociale et économique que connaissaient ou connaissent encore certains dirigeants arabes et leurs régimes. Par conséquent, le pouvoir et ses institutions se trouvent confrontés à un processus de récupération de cette légitimité sociopolitique qu'ils ont perdue (Almanjdra, 2003)

Ce duel entre la reprise de la légitimité et la délégitimation, traduit par des révoltes, des manifestations et des protestations, dont certaines ont provoqué une guerre civile comme dans le cas de la Libye, est aussi un combat discursif. Une bataille où l'accès au discours de légitimation des opinions, des événements et des actions a été et demeure toujours un élément clé dans ce processus. Ainsi, l'utilisation des pratiques discursives à travers l'accès au discours politique du pouvoir ou le discours des médias ou des réseaux sociaux a été un facteur important dans l'échec, le succès ou la continuité des ces deux processus : la récupération de la légitimité sociopolitique par le pouvoir et la délégitimation des appareils de l'État par les acteurs sociaux protagonistes de ces mobilisations populaires.

De ce fait, nous estimons qu'il convient d'analyser à partir d'une approche critique de l'analyse du discours (CDA) ces processus sociopolitiques de légitimité, et surtout la délégitimation du pouvoir de l'État et de ses actions, ses politiques sociales et économiques. Notre recherche portera ainsi sur le Mouvement 20 Février au Maroc et son discours.

L'objectif principal sera l'analyse des représentations sociales du pouvoir et de soi-même comme stratégie socio-discursive utilisée dans tous les genres discursifs produits par ce Mouvement. Les communiqués de presse, les manifestes, les interviews avec des militants activistes, les vidéos sur Youtube et les slogans répétés durant les marches composeront notre corpus linguistique.

\section{Pourquoi l'Analyse critique du discours?}


Les recherches sur les mouvements de contestations sociales et politiques peuvent être envisagées sous différentes perspectives disciplinaires (historique, politique, juridique, économique, sociologique, linguistique), chaque discipline ayant sa propre tradition et son propre cadre théorique et méthodologique. Toutes ces approches et ces disciplines doivent toutefois tenir compte d'un même élément fondamental, le discours, sous toutes ses formes, écrit, oral, ou autre.

Suivant la notion théorique d'Analyse critique du discours, considérée parmi les approches qui reposent sur une synthèse des études critiques portant sur le changement social dans la société contemporaine, le discours fait partie intégrante du social, et en tant que tel, forme et est formé par celui-ci. De cette manière, tout genre de discours est considéré comme une utilisation de la langue, une représentation sociale, une communication des croyances et une interaction, chacune de ces quatre dimensions étant reliée aux autres.

A partir de cette notion, l'intérêt autour du discours partagé entre les sciences humaines et sociales n'est pas dissocié de l'interprétation de l'activité discursive comme une pratique. Autrement dit, c'est une activité qui est socialement régulée et qui dispose d'une notion historique et dynamique en même temps, celle du discours comme pratique sociale, discursive et textuelle. Cette conception du discours qui prévaut parmi les autres pratiques dans le CDA, fait que les préférences d'analyse sont dirigés vers non seulement ce que dit le discours, mais ce qu'il fait ou est en mesure de faire. Tout cela, nous amène à analyser les effets sociaux et politiques des discours et les différentes valeurs sociopolitiques et idéologiques qui leur sont attribuées dans le champ discursif.

\section{Cadre théorique et méthodologique}

A partir de ces caractéristiques, nous suivrons deux lignes de recherche qui nous intéressent dans cette approche critique de l'analyse du discours: premièrement, l'étude et l'analyse de la façon dont les discours sont organisés et ordonnés, et comment ils renferment une interprétation des faits et de la société ; la deuxième ligne de recherche est celle qui considère le discours comme une manière de signifier un domaine particulier de la pratique sociale. Ainsi, nous concevons le discours comme une série d'événements, de formes et d'usages langagières (Wodak, 2003), voire un ensemble de stratégies discursives qui sont énoncées afin de faire circuler des savoirs concernant des acteurs de la société, des événements et des actions, et de les légitimer sur le plan social et politique.

Une définition générale et globale qui pourrait définir la notion de Contexte, dans le CDA, est celle proposée par Van Dijk (1999, p. 266) : "l'ensemble structuré de toutes les propriétés d'une situation sociale qui est potentiellement pertinente pour la production, les structures et les fonctions d'interprétation d'un texte et d'une conversation ". De plus, Van Dijk, (op. cit) considère comme essentielle pour briser, distinguer et enrichir cette définition globale du contexte, une notion de contexte local défini comme l'ensemble des caractéristiques de la situation immédiate et interactive où l'acte de parole a eu lieu. Selon Van Dijk, (2003, p. 160), ce modèle de contexte est directement lié aux structures de la parole parce que l'auteur fait également une distinction entre le sens et la signification globale et locale, et entre formes discursives globales et d'autres 
locales. Au sein de cette notion de contexte, nous allons donc essayer d'expliquer le contexte des discours de notre corpus. En premier lieu, nous présentons le contexte global actuel des discours que nous analyserons, un savoir qui correspond au contexte historique, en l'occurrence tous les événements politiques et sociaux qui ont eu cours au Maroc et dans certains pays de la région, dont la Tunisie et l'Égypte et qui encadrent d'une certaine manière la naissance du M20F. Ensuite, nous avançons une analyse détaillée du contexte local des discours analysés du M20F, à savoir, le contexte immédiat de chaque acte de communication.

En ce qui concerne l'analyse des processus de légitimation et délégitimation, nous partons de la notion de légitimité discursive (Van Dijk et Martin Rojo, 1997 ; Van Dijk, 2003 ; Chilton 2009). Par ailleurs, nous nous appuyons sur les stratégies discursives (Wodak et autres 1999, Wodak 2001 ; Wodak, 2003) en examinant les stratégies discursives de justification et de légitimation. Deuxièmement, pour l'analyse de ces stratégies, nous nous concentrons particulièrement sur les structures des textes utilisées dans les discours comme des stratégies de référence. Ces stratégies seront i) les traits qui au M20F de s'identifier et quels rôles jouent ces traits dans le processus d'autoreprésentation et de construction de cette légitimité sociale et politique que cherche le M20F, et en même temps son désir de délégitimer les actions sociales et politiques mises en place par l'État et ses appareils ; ii) l'analyse des formes de dénomination du pouvoir et de l'État utilisées par le M20F et la valeur sociale attribuée à ce pouvoir de l'État et sa relation avec les actions et les processus qui ont plus de pertinence dans le discours du M20F.

\section{Les discours de résistance et les mobilisations sociales au Maroc: un nouveau champ de recherche}

Nous partageons avec Ben Néfissa (2011, p. 77) l'idée qui indique le caractère littéraliste dans un modèle d'interprétation des systèmes autoritaires arabes et des processus de réactualisation du pouvoir politique. L'une des conséquences de cette notion littéraliste, comme l'a bien expliqué l'auteur, est de " renforcer l'illégitimité de l'objet "mouvement social » dans les études portant sur la région [...] conduisant la recherche à ne pas prendre aux sérieux les protestations sociales et économiques qui ont précédé les deux révolutions ", c'est á dire la tunisienne et l'égyptienne (op. cit, p. 83). L'absence des études concernant le discours des mouvements sociaux de contestation, plus exactement au Maroc, est l'une des raisons pour lesquelles nous nous sommes engagé à réaliser cet article. Même si il existe des recherches et des études menées jusqu'à maintenant à partir des approches sociologique, historique ou politique sur les mouvements sociaux de contestation, notre objectif dans ce travail est d'intégrer une théorie sociale du discours dans l'analyse du " mouvement social » comme objet de recherche. L'intégration du CDA dans la recherche sur le mouvement social est justifiée aussi par le fait que les mouvements sociaux aux Maghreb, plus concrètement au Maroc, ont subi un processus dynamique de changement qui les a conduits vers ce que Ben Néfissa (op. cit, p. 5) appelle « Hybridation du politique et mouvement social ». Ce caractère hybride nous permettra de comprendre l'évolution des pratiques discursives des mouvements de contestation vers : a) un discours politique plus 
organisé et plus clair au niveau de sa structuration, et b) une disciplinarisation de son activisme en général et de sa confrontation avec le pouvoir publique. De la même manière, cette hybridation nous amène, d'une part, à prendre en considération le contexte politique, social, économique et idéologique où émerge le discours des mouvements sociaux au Maroc, plus précisément du Mouvement 20 Février (M20F). D'autre part, une analyse de ce genre nous oblige aussi à situer le M20F dans un micro-contexte qui correspond évidement à toutes les formes de protestation, sociale, politique, linguistique ou identitaire qui existent actuellement dans le champ sociopolitique et discursif au Maroc. Enfin, l'analyse d'un discours social et politique portant sur des acteurs et des groupes sociaux opposés au pouvoir et avec ce qu'on appelle les discours de résistance, enrichira cette théorie sociale située dans l'Analyse critique du discours.

\section{Analyse du contexte historique et sociopolitique au Maroc et émergence du Mouvement 20 Février}

La conception de la politique qu'avait l'État marocain, depuis l'indépendance jusqu'à l'an 2000, est à plusieurs égards différente de ce qu'elle est de nos jours. Les changements initiés par le jeune roi Mohamed VI depuis son accession au trône en juillet 1999 ont introduit évidemment une nouvelle façon de penser la société, du moins pour les acteurs des mouvements sociaux, une nouvelle façon de penser l'économie et une nouvelle façon de penser la démocratie et les droits humains, même si cet éveil ne s'est pas étendu à toutes les composantes de la société marocaine. En effet, le Maroc a connu et connaît toujours des dynamiques de changement sociale et identitaire qui exercent une influence sur les politiques de l'État et ses appareils; cette influence est conçue comme une option pragmatique plutôt que symbolique et idéalisée autour de l'action publique sur le politique en général. Pour cette raison, nous concevrons l'analyse du contexte où émerge le M20F dans la perspective des dynamiques sociopolitiques, économiques, territoriales et identitaires en cours, en mettant l'accent sur les changements et les actions qui ont eu lieu durant cette dernière décennie (voir Bennfla, 2011, Catusse, et Vairel, Moustaoui 2011). L'analyse du contexte sera envisagée aussi dans une approche critique.

\section{Dix ans de réformes et $\mathbf{1 0}$ ans de protestations: où se dirige le Maroc?}

Derrière un discours officiel qui annonce " une nouvelle notion d'État», on peut situer les différentes initiatives à caractère sociopolitique menées par le pouvoir depuis l'année 2000 jusqu'à nos jours. Le lancement de l'Initiative nationale pour le développement humain (INDH) à travers la mise en place de nouveaux dispositifs institutionnels dont le but était articuler l'action publique et les mobilisations des collectives, soit local ou régional a été accompagnée par une série de reformes législatives concernant Le Code de la famille (la nouvelle Moudawana), Le Code du travail, et Le Nouveau code de la route, etc. ; de même, il faut souligner, sur le plan judiciaire, la création de la Commission Vérité et Réconciliation prenant le nom après de l'Instance équité et réconciliation (IER). L'INDH a pour objectif également de lutter contre l'accroissement des bidonvilles et de freiner l'immigration interne vers les villes 
et les métropoles ainsi que l'immigration clandestine vers l'Europe. Sur le plan économique, cela a donné lieu à la multiplication des grands chantiers en matière de transport et d'infrastructure urbaine et rurale. Dans la même ligne imposée par le néolibéralisme économique, le Maroc a suivi les recettes des grands multinationaux. Cela se traduit par l'implantation des grands projets immobiliers, surtout avec la crise économique en Espagne et en Italie, la construction des novelles zones industrielles, des nouveaux parcs scientifiques et des hubs financiers à Casablanca, Tanger, Marrakech, Fès et Agadir. Ces actions ont encouragé évidemment les flux touristiques, attiré des nouvelles formes d'investissements au Maroc et surtout favorisé l'augmentation des transferts d'argent des Marocains résidant à l'étranger (MRE) atteignant en 2009 le 6\% du Produit national brut (Bennafla, op, cit).

Le champ linguistique et identitaire marocain, caractérisé historiquement par une stratification sociale et politique, a pour sa part bénéficié des changements initiés par l'État. La reconnaissance du composant amazigh de l'identité nationale marocaine - unique, singulière et unifiée - dans le discours d'Ajdir en Juillet 2001 et la création de l'Institut Royal de la Culture Amazigh sont autant de preuves qui témoignent d'un désir de l'État marocain d'acquérir ce pluralisme démocratique durable (Boukous, 2008). Dans le même cadre, nous rappelons toutes les initiatives visant la reconnaissance et la préservation du pluralisme marocain. Un pluralisme dont les atouts demeurent une diversité linguistique historiquement attestée, et une diversité identitaire, le tout représentant le ciment de cette nation. L'État se trouve obligé de redéfinir ses positions et ses représentations de ce pluralisme dont les défenseurs exercent de plus en plus de pression et remettent en question son modèle de gestion de cette diversité. Le projet de la Nouvelle Constitution de 2011 et la régionalisation avancée sont des témoignages clairs de ces mutations au niveau des pratiques discursives de la monarchie et de ses institutions. II n'en demeure pas moins que dans ses représentations de l'identité l'État insiste toujours sur l'existence d'une seule identité nationale unie et homogène.

La question qui se pose, et que nous partageons avec Catusse et Vairel (2011, p. 8) est la suivante : En quoi le régime marocain se transforme t-il ? Toutes les réformes sont, reconnaissons-le, insuffisantes et lentes. «Au Maroc, le « printemps arabe » invite à s'interroger sur la signification de la réforme, sur ce que réformer veut dire. Sur ce sujet, les pistes de réflexion sont innombrables » (Hibou, 2011, p. 3). Il est sûr, par ailleurs, que la mobilisation des acteurs sociaux ne faiblit pas face au manque d'une réelle volonté de transformation de la part de l'État. De la même manière, les discours de résistance émergent quotidiennement remettant en question les réformes et les actions mises en place par l'État dans tous les domaines. Du point de vue des contestataires, les réformes entreprises ne seraient qu' "un double désamorçage " puisque d'après eux «les mutations qu'a connues la société marocaine n'avaient jusqu'à présent pas trouvé d'expression institutionnelle qui puisse donner une idée de l'orientation que le régime allait prendre par rapport à elles : allaientelles s'inscrire, oui ou non, dans un tournant résolument démocratique? " (Ferrié et Dupret, 2011, p. 29).

Mais au-delà de ces interprétations basées sur une analyse des données, la réalité socioéconomique du Maroc n'est pas des plus enviables. Rappelons que les diagnostics sur l'état social sont alarmistes. En plus, Le Maroc est parmi les 
pays arabes les plus mal classés sur l'échelle de l'indice du développement humain, selon le Programme humain des nations unies pour le Développement (PNUD) ${ }^{1}$. Le taux de chômage est élevé. Au niveau de l'éducation, durant la dernière décennie, l'État a mis en place une politique de privatisation de l'enseignement public. Cette politique a provoqué une détérioration du système éducatif marocain et un échec du programme d'alphabétisation (en remontant l'indice multidimensionnel de pauvreté). II faut noter aussi la situation du système sanitaire quasi inexistant et l'état de l'administration chargée de la justice. Ces trois derniers domaines ne bénéficient d'aucune intervention directe ou de quelconque mesure d'amélioration. Enfin, nous devons signaler l'absence d'une vision claire sur le processus de la mise en place des recommandations de l'IER. Ce qui explique l'importante multiplication des associations qui défendent l'établissement urgent de bases solides d'un État démocratique et de droit. Nous pensons au rôle que joue l'AMDH, l'organisme le plus actif dans le militantisme qui défend les droits de l'homme au Maroc.

Parallèlement à cette vague de réformes institutionnelles que nous estimons trop pauvre, des mobilisations sont initiées depuis près de 10 ans. Le fil conducteur dans le discours de ces protestations était toujours l'élargissement de l'espace de participation et de discussion. Cette présence forte d'un activisme civil issu de la société civile présente le Maroc comme " l'avant-garde de la protestation sociale dans la région »(Catusse, 2011). On peut décrire cette situation comme une espèce de résistance ayant créé, en terme socio discursif, un dialogue caractérisé par la confrontation et le conflit. Un conflit dont la principale raison est le modèle de l'État mis en place pour la gestion des biens matériels et symboliques ${ }^{2}$.

Cet activisme civil a ainsi pris différentes formes. Nous commençons par le Mouvement Culturel Amazigh qui est de fait le Mouvement et le discours le plus ancien qui revendique un modèle de gestion et de gouvernance différent et opposé en quelques termes à celui de l'État. Un mouvement qui a connu une évolution dans ses stratégies de résistance et qui n'a jamais été satisfait de ce que l'État a réalisé jusqu'à présent au niveau de la gestion politique et sociale de la question amazigh, celle-ci étant une question de caractère non seulement sociolinguistique et identitaire mais aussi économique et territorial.

S'inscrivent dans cette perspective toutes les associations issues de l'université comme l'Union nationale des étudiants marocains (UNEM) et l'Association Nationale des diplômés chômeurs au Maroc (ANDCM), créée en 1991. Nous citons ces trois mouvements en exemple parce qu'ils jouissent d'une présence et d'une visibilité au sein du champ universitaire, social, politique, médiatique et discursif au Maroc. Nous pensons qu'ils ont réussi à changer en quelque sorte la façon de penser l'activisme civil en défiant les formes de répressions exercées par l'État, d'une part; et en transformant, d'autre part, le dialogue

\footnotetext{
${ }^{1}$ D'après le rapport annuel de la PNUD 2010, le Maroc occupe le 114 rang derrière la Tunisie, l'Algérie et la Libye.

2 D'aprés (Ben nefissa, 2011, p. 15) « L'expression mujtama' madani (société civile) fait partie du langage et «l'au-nom- de-la-société-civile » légitime non seulement des discours multiples, mais aussi des actions et des pratiques. Ces effets de réel sont d'autant plus importants qu'ils sont l'objet d'enjeux de taille en rapport avec l'aide internationale à la société civile. Mais ils recouvrent également des enjeux symboliques, économiques, politiques, religieux et identitaires sur le plan interne (Otayek, 2002 ; Pirotte, 2007) ».
} 
discursif le rendant plus ouvert à la participation et à la circulation des nouvelles stratégies de représentations du modèle et des actions revendiquées concernant le politique au Maroc. Dans ce sens, à partir de 2006, on constatait la création des coordinations locales qui dénonçaient la hausse des prix et la dégradation des services publics dans plusieurs villes et villages. L'activisme à travers ces coordinations locales a adopté comme forme de protestation l'organisation de différentes marches nationales et Sit-in qui ont permis d'obtenir des résultats positifs. Rappelons aussi les contestations sociopolitiques qui émergeaient dans la région du Sahara en lien avec la question de l'autonomie et de l'indépendance; elles se basaient sur des arguments géopolitiques, sociohistoriques et linguistico-identitaires. Les événements qui ont eu lieu dans le camp de "Agdim Izik» à Laâyoune en novembre 2010 est une excellente épreuve.

C'est à cause de cette vague de protestations que l'espace d'activisme civil au Maroc est devenu de plus en plus hétérogène. Le processus de modernité et les mutations socioculturelles, démographiques et médiatiques jouaient et jouent un rôle très important dans l'apparition de nouveaux acteurs sociaux. Dans ce cadre, il faut souligner la naissance de nouveaux mouvements de mobilisations sociales. Ces mouvances ont crée en même temps des espaces publics $^{3}$ où émergent de nouvelles pratiques discursives et linguistiques revendiquant des droits et des libertés individuelles. C'est le cas du mouvement Nayda à Casablanca basé sur une initiative locale, et plus précisément le quartier L'Boulevard dont l'objectif est de défendre une forme de liberté avec l'utilisation des expressions artistiques et musicales en arabe marocain. Dans cette ligne qui défend les libertés individuelles, il convient d'évoquer le Mouvement alternatif pour les libertés individuelles (MALI). C'est une mouvance née aussi sur Facebook en août 2009; elle est l'œuvre de deux jeunes marocaines : Zineb Rhazaoui et Ibtissam Lachger. L'objectif de ce mouvement était de discuter de la question des libertés individuelles, en particulier, la liberté de culte, d'expression, de sexe et la liberté de vie professionnelle. Dans un appel à mobilisation annoncé sur Facebook, le mouvement déclare : "Nous prônons la liberté d'opinions, de croyances et de choix de vie personnels. Nous lançons un appel à un rassemblement solidaire permettant la restructuration d'une société de solidarités et de garanties effectives des libertés individuelles " (Sidi Hida, 2011, p. 174). Le mouvement a eu sa popularité surtout grâce a cet appel de "Ouaklin Ramdan », Dé-jeûners de Ramadan. Même si ces actions sont perçues par l'État et une partie de la société uniquement en relation avec le facteur de liberté de culte et de rupture de jeûne, MALI a convoqué aussi des rassemblements qui n'ont rien à voir avec le Ramadan. En mai 2010 a eu lieu un sit-in à Casablanca pour réclamer la liberté sexuelle et dénoncer le harcèlement sexuel dont sont victimes des femmes. Le mouvement est toujours présent sur Facebook et son activisme délaisse progressivement son aspect virtuel au profit d'un militantisme de fait et d'actions.

Un autre mouvement basé aussi sur la défense des libertés individuelles c'est le mouvement KifKif LGBT qui regroupe Lesbiens, gays, bi et transsexuels du

\footnotetext{
${ }^{3}$ Nous utilisons ici la notion « d'espaces publics » défini par Ben Néfissa (2011, p. 86) comme " espaces symboliques de délibération, et non de décisions, dans lesquels les individus privés échangent et communiquent sur des questions d'intérêts généraux, dégagent des opinions publics. »
} 
Maroc. C'est un mouvement né en 2004 sur Facebook; il a comme objectif de sensibiliser la société marocaine à la question des droits individuels des homosexuels. Les deux mouvements, MALI et KifKif, reflètent l'importance des espaces sociaux et virtuels dans les mobilisations sociales. Ceci encourage la création de nouvelles plateformes de blogosphères. Des blogs ont ainsi vu le jour dont Blogger.com et Maktooblog.com. Youtube et Dailymotion se sont également avérés être des outils aussi efficaces. Au sein de cette blogosphère surgissent des opinions, des débats et des critiques autour du changement social et politique au Maroc (A-taghyir التغيير) et autour des représentations sociales et du rôle de l'État dans ce processus de transition. Tout ceci constitue l'axe central autour duquel le M20F va légitimer sa naissance et va construire son discours pour s'imposer comme une mouvance qui représente presque la première force d'opposition au Maroc.

\section{L'émergence du Mouvement 20 Février}

La naissance du M20F est indissociable du contexte sociopolitique du monde arabe et de celui du Maghreb en particulier après le succès de la révolution tunisienne. Même si l'approche de l'Analyse critique de discours met l'accent sur l'analyse du micro-contexte comme introduction à l'analyse linguistique des données, les événements qui se sont déroulés dans la région, Maghreb et Moyen Orient, jouaient un rôle fondamental dans la naissance de ce mouvement et dans la direction qu'il prendra par la suite pour avoir cette légitimité sociopolitique et discursive. Rappelons que l'expérience tunisienne a commencé par des mobilisations et des soulèvements sociaux dans les zones déshérités. La naissance d'un mouvement de contestation populaire au Maroc, suite aux succès de la révolution tunisienne, pourrait être interprétée comme un fait prévu.

Le 20 Février est la date qui marque l'apparition du M20F qui s'est clairement inspiré de la Tunisie et de l'Égypte sans pour autant toutefois viser le renversement du régime. Le mouvement se définit comme « une force de protestation et non de proposition ${ }^{4}$.» «Indépendant de tout parti politique, syndicat et autres organisations. Le mouvement ne s'essouffle pas, il se propage de la rue vers les institutions, les partis ${ }^{5}$." Son organisation lui donne une puissance parce qu'il ne dispose pas de leaders ou porte- parole. De même, le mouvement n'a pas d'identité unifiée. De 30 coordinations dans tout le royaume, il est passé à 115 entre la date de sa naissance et le 24 avril. Le Mouvement rassemble en son sein des forces de progrès : L'AMDH, organisation qui s'aligne ouvertement sur les objectifs du M20F dès sa naissance; partis de gauche comme La Voie Démocratique et le Parti Socialiste Unifié (PSU) ; jeunesse, société civile, mouvements de femmes, mais

\footnotetext{
${ }^{4}$ Interview avec Elabidila Chbina, activiste du M20Fde la cellule de Rabat, Magazine Tel quel, $n^{\circ} 473,2011$.

${ }^{5}$ Interview avec Aadel Es-Saâdani, activiste du M2OF de la cellule de Rabat, Magazine Tel quel $n^{\circ} 473,2011$
} 
aussi islamistes, en l'occurrence Al-'adel Wa Al-lhsane (Justice et Bienfaisance) $)^{6}$.

Avec le succès de la première manifestation, le M20F réussit à faire renaître la culture des manifestations au Maroc. De cette manière, il s'est imposé dans la scène politique et associative nationale. Dés sa naissance jusqu'à maintenant le M20F a convoqué plus d'une dizaine de marches au niveau national, avec la moyenne d'une marche par mois, sans compter les marches, les rassemblements et les Sit-in convoqués par les coordinations locales dans chaque ville. De même, le M20F connait une internationalisation à travers la création de cellules à l'étranger. II a ainsi gagné en popularité dans le cercle des Marocains militants à l'étranger. C'est en France que le M20F a plus de coordinations puisqu'il' y a presque une dizaine de cellules partout en France; on trouve des cellules également aux Pays-Bas, aux États-Unis, au Canada et en Espagne.

De manière générale, on peut dire, reprenant l'idée de Bennafla (2011, p. 16), que le M20F se distingue du reste des mobilisations qui ont émergé au Maroc par les traits suivants : " la politisation ouverte du cahier revendicatif qui tranche avec la politisation en creux des précédentes, [...] le second point original est la coordination à l'échelle nationale d'actions protestataires dispersées grâce à l'utilisation des réseaux sociaux ॥. Et le troisième trait est lié à l'influence et au pragmatisme exercés par le M20F sur l'ordre discursif et sociopolitique au Maroc. Son discours a dès le départ bénéficié de beaucoup d'estime dans le discours officiel dominant quoique de manière indirecte. Et c'est ce que nous allons traiter dans le point suivant à travers une analyse de l'ordre discursif qui s'est établi au Maroc avec l'émergence du M20F.

\section{Ordre discursif avant et après la naissance du M20F}

Nous sommes d'accord avec l'idée proposée par Cicourel (1980) qui affirme l'existence d'une relation directe entre la manière dont le pouvoir est distribué et la structuration du champ du savoir. Nous concevons la connaissance telle qu'elle a été définie par Heller (2007, p. 635) « as organized sets of discourses with organic connections among each other that take shape as a function of how institutional processes are organized and how actors are involved in the production and circulation of resources. I will use the notion of trajectory and of discursive spaces ». A partir de cette définition, il faut souligner qu'il existe au Maroc un ordre discursif et un champ de savoir caractérisés par une forte domination des rapports de force où les discours officiels (monarchiques et législatifs) sont considérés comme des discours et des espaces dominants dans un ordre social et politique complètement stratifié et hiérarchisé (Foucault, 1979 ; Bourdieu, 1982). Mais ceci ne veut pas dire qu'il n'existe pas d'autres espaces discursifs avec des représentations sociales différentes et même opposées à celles de l'État.

Dans ce sens, les événements qui ont présidé à la naissance du M20F ont exercé une influence directe sur l'ordre du discours et sur les représentations

\footnotetext{
${ }^{6}$ Le 19 décembre 2011, Le mouvement Al-'adel Wal Ihssane a annoncé dans un communiqué sa décision d'arrêter de manifester dans le cadre des marches du M20F, malgré leur conviction de la légitimité des revendications de ce mouvement.
} 
sociales et politiques au sein du discours officiel même. Si l'émergence du M20F a joué un rôle essentiel dans la configuration d'un nouvel ordre discursif, quels sont les aspects qui nous démontrent qu'on est vraiment en présence d'une nouvelle configuration dans cet ordre ? Quels sont les nouveaux espaces discursifs qui utilisent le M20F pour faire circuler son modèle de connaissance et ses représentations sociopolitiques ? Et comment a été configuré ce dialogue discursif entre l'État et le M20F avant et après le 20 février 2011.

Statistiquement, le nombre de discours qui ont été prononcés par le roi durant les deux dernières années concernant des réformes institutionnelles, a été considérable. Nous pouvons dire que cette explosion discursive monarchique a déjà commencé dés le 3 janvier 2010, date du discours royal qui établit la création d'une commission consultative sur le projet de régionalisation avancée. Dans ce discours, le roi annonce:

« Nous entendons également en faire un prélude à une nouvelle dynamique de réforme institutionnelle profonde ».

Ce discours est une réponse aux événements qui ont eu lieu à Laâyoune dans le camp de "Agdim Azik » juste deux mois avant sa prononciation. L'objectif de tout discours dominant évidement est d'exercer un contrôle social sur les masses et de renforcer la légitimité idéologique et politique de ses appareils. Dans cette même démarche de réformes, cette fois-ci c'est l'ex premier ministre Abass Al Fassi qui annonça le 20 janvier 2011 la création de 1000 postes d'emploi pour des chômeurs diplômés et la réservation du $10 \%$ des nouveaux postes pour les diplômes de troisième cycle.

Mais un mois après, c'est le discours de 20 février qui s'impose et occupe deux espaces discursifs que l'État n'a jamais osé exploiter : la rue et l'espace virtuel à travers les différents réseaux sociaux. Les intentions réformistes de l'État ne sont pas très loin des intentions du M20F, car selon les vidéos apparues sur Youtube et qui annoncent sa création, elles intègrent des revendications connus dans le milieu associatif marocain :

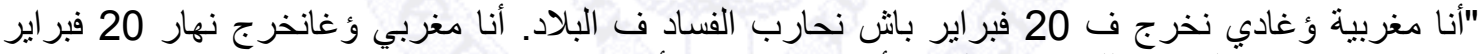

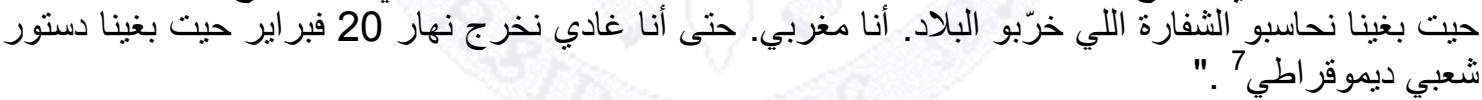

" Je suis marocaine. Je vais sortir le 20 février pour lutter contre la corruption qui existe dans le pays. Je suis marocain. Je vais sortir le 20 février parce que nous voulons rendre devant la justice tous ceux qui ont volé et détruit le pays. Je suis marocain. Moi aussi je vais sortir le 20 février car nous voulons une constitution issue du peuple et démocratique $»$.

Rien de nouveau au sein des revendications du M20F. Néanmoins, les arguments qu'il met en avant font le lien entre la détérioration des services publiques et l'aggravation de la corruption, sans oublier évidement le taux élevé du chômage parmi les jeunes marocains, sachant que la majorité des militants de ce mouvement est âgé entre 18 et 35 ans. De même, pour imposer sa légitimité, le M20F a repris à son compte un élément clé : la relation historique et réciproque qui existe entre la précarité matérielle et l'exercice de l'autoritarisme officiel de l'État.

\footnotetext{
${ }^{7}$ Vidéo première compagne qui annonça la création du M2OF et fait appel aussi à la première marche organisée par le mouvement.
} 
Mais, dans cette même logique de renforcement de la légitimité et de la crédibilité, le roi annonce dans le discours prononcé le 21 février 2011, un jour après l'émergence du M20F, l'installation du conseil économique et social. Cette démarche s'inscrit d'après le roi dans le cadre suivant:

«Une forte impulsion à la dynamique réformatrice que Nous avons enclenchée dès que Nous est échue la mission d'assurer la conduite de Notre peuple fidèle. [...] la poursuite de la réalisation des réformes structurantes, suivant une feuille de route dotée d'une vision et d'objectifs clairement définis, et fondée sur l'étroite symbiose entre le Trône et le peuple. Notre but ultime est d'assurer à tous les Marocains les conditions propices à l'exercice d'une citoyenneté digne, dans le cadre d'un Maroc avancé, solidaire et jouissant de son unité et de sa souveraineté pleines et entières $"$.

D'ailleurs, cette dynamique discursive du monarque reste toujours liée à ses intentions réformistes; de même, il ne soutient pas l'idée de transition dans la mesure où les réformes devraient être envisagées comme un changement dans le régime qui garantit ipso facto le remplacement des institutions et les élites de l'État par d'autres choisis par le peuple.

Le 9 mars, 20 jours après la naissance du M20F, le roi décida de prononcer un autre discours dans un contexte dominé par l'actualité des révolutions arabes. Le roi annonça ainsi la préparation d'une nouvelle constitution, adoptée le premier juillet 2011 après un référendum, et en partant encore une fois de ses intentions réformistes et suivant la même ligne précédente. Peut-on dire que le roi a réagi ? II n'y a pas de doute que c'était une réaction face aux événements qui marquaient l'actualité nationale et internationale. Nous pouvons considérer et interpréter ce discours de deux manières : la première est que le roi utilise l'accès au discours pour freiner et créer une délégitimation des revendications et une dépolitisation du M20F. C'est-á-dire que le discours du monarque désamorce cette crédibilité, sociale du moins, acquise par le M20F. La deuxième interprétation va dans le même sens que la première dans la mesure où le roi maintient son statut dans cet autoritarisme d'État et confirme ainsi la relation qui existe entre les rapports de force au niveau des pratiques discursives dominantes et les actions qui suivent, sachant que cette fois il s'agit d'une réforme majeure de la constitution.

Mais le discours de 9 mars a donné un souffle nouveau au M20F qui le considère comme un discours conservateur qui passe sous silence l'essentiel du pouvoir royal. Ce mécontentement du M20F a été traduit par la convocation d'un Sit-in qui a été dispersé le 13 mars devant le siège du PSU. Mais le 20 mars, le M20F organise une grosse manifestation nationale pour annoncer son désaccord avec la réforme constitutionnelle du 9 mars. Dans les vidéos qui annonçaient la manifestation du 20 mars et les nouvelles revendications, le M20F affichaient clairement son point de vue sur la réforme de la constitution :

$$
\text { "بغينا دستور شعبي جديد. المخزن بغا غير يرقع ديالو القديم. حنا راه ما مفكينش } 8 \text { " }
$$

« Nous voulons une constitution issue du peuple et démocratique. Le Makhzen veut seulement rapiécer son ancienne constitution. Mamfakinch (Nous ne lâchons pas)».

\footnotetext{
${ }^{8}$ La vidéo qui annonce la création du M20F lance aussi l'appel à la première marche organisée par le mouvement.
} 
C'est à partir de ce moment qu'a commencé la bataille discursive. Dans cette manifestation du 20 mars, le M20F a montré clairement son refus du nouveau projet de constitution comme le montrent les slogans répétés dans la rue : اسمع "لاسع "لا للفصل 19 من Sma'a Sawt A-cha'b » (écoute la voix du peuple) ou صوت الثعب" "La li Al fasel 19 mina A-doustour» (Non à l'article 19 de la constitution). La stratégie de communication du M20F est basée sur la production et la reproduction d'un discours issu du peuple et pour le peule. En plus, la plateforme Mamfakinch est considérée comme l'arme de

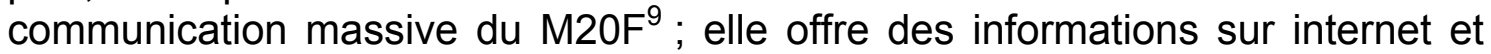
les réseaux sociaux. Tout cela a donné de bons résultats au niveau de l'impact de ce discours sur les masses et l'émergence d'un modèle alternatif de représentation de l'État et de ses actions.

Un mois après la manifestation du 20 mars, le M20F convoqua le 24 avril le troisième grand rassemblement. Cette fois le discours vise directement le roi et l'un des slogans répétés était " Allah, Al watan, Al Houriya wa Al-Karama " (Dieu, Patrie, Liberté et Dignité). De même, dans la manifestation nationale du 22 mai, la représentation du roi est de plus en plus différente de celles d'avant. Cette fois les slogans franchisent les limites et s'articulent sur la chute du régime "Acha'b yourid Isquat A-nidam » (Le peuple veut la chute du régime), et aussi sur la transition vers un régime républicain "بغيتو الحرية قلبو ها جمهورية" "Ila Bghitou Al Houriya, qualbouha Jamhouriya » (Si vous voulez la liberté il faut la transformer en république). Cette manifestation a été marquée par une violente intervention des forces de l'ordre qui s'appuyaient sur la non-autorisation des marches par l'État. Le mouvement s'est engagé dans un bras de fer avec le pouvoir; son discours donne alors les signes d'une sorte de radicalisation dans le mouvement. Khalid Naciri, ex-porte-parole du gouvernement a affirmé le lendemain de la manifestation du 22 mai :

« Tant qu'on avait affaire à des jeunes qui réclament des réformes démocratiques, il n'y avait aucun problème. Mais là, nous sommes face à une autre configuration ».

Dans ces circonstances, des groupes anti-M20F organisaient des manifestations et des marches en faveur d'un changement mais par le roi, avec le roi et en insistant sur la sacralité des valeurs de la monarchie marocaine: « Dieu, Patrie et Roi ». Mais dès le dernier discours du 9 mars, le roi décida de ne pas se prononcer. En même temps, le M20F devait repenser ses stratégies de mobilisation et rendre son discours réaliste. Cela signifiait informer plus et se rendre plus proche des autres classes de la société, à savoir, les couches sociales pauvres. Dans cette ligne, Omar Radi, un des militants du M20F affirma :

« Nous avons remarqué que la perception de l'opinion publique a changé à notre égard. Cela n'est pas nullement dû à une prétendue radicalisation du mouvement, mais surtout à la campagne de désinformation menée par les services de l'État ${ }^{10} . "$

\footnotetext{
${ }^{9}$ Voir l'article d'Aisha Akalay paru dans le no 479 du Magazine Tel-quel, « Mamfakinch. Arme de communication massive $"$.

${ }^{10}$ Interview avec Omar Radi, militant du M20F, apparu dans Magazine Tel-quel, no 477.
} 
Le M20F était obligé de se déplacer vers les quartiers populaires et créer des coordinations locales dans différentes villes du Maroc ${ }^{11}$. Il faut souligner aussi que sa force de mobilisation s'est réduite à cause des autres rassemblements et marches pro-régime et anti-M20F.

Le 17 juin 2011, deux jours avant le lancement de la campagne pour le référendum sur la nouvelle constitution, le roi prononça un discours où il insinua que voter OUI est une solution pour les problèmes du Maroc. Un discours qui construit discursivement et socialement un « Nous » formé par l'État, le pouvoir et le peuple et un "eux" composé par les opposants à ce projet de constitution. Le « oui » du roi évoque évidement l'existence d' " un non »; ceci révèle une confrontation sociopolitique et idéologique mais dépolitisée par le roi. Ce qui s'est passé lors de la manifestation du 26 juin le reflète parfaitement quand opposants et partisans au projet de la nouvelle constitution se sont rencontrés dans la rue. Mais, il fallait attendre le 31 juillet, fête du Trône, qui coïncide toujours avec la prononciation du discours du roi dont le contenu est d'une grande importance pour l'État et même pour le peuple. Dans ce discours le roi annonça ce qui suit :

« Mais toute Constitution, aussi parfaite qu'elle puisse être, n'est ni une fin en soi, ni même le terme d'un parcours. Elle constitue plutôt une base solide pour un nouveau pacte constitutionnel marquant la volonté d'aller de l'avant dans la mise en place d'institutions efficientes et crédibles, en vue de la consolidation de l'État de droit et des droits de l'Homme, de la bonne gouvernance et du développement. [...]Parallèlement, il faudra veiller à rendre effective la consécration constitutionnelle du rôle assigné à la société civile et aux médias dans la construction politique et dans les domaines des droits de l'Homme et du développement. Ils devraient ainsi pouvoir assumer efficacement leurs responsabilités en tant que force de proposition, et comme levier efficient et partenaire fondamental dans le processus de consolidation de cette construction $»$.

D'une part, le nouveau projet de constitution s'inscrit selon le roi dans un long processus dont la société civile est aussi responsable. Et, d'autre part, aucune mention au M20F n'a été faite. Par contre, on trouve toute une série de stratégies discursives dont le but principal était de légitimer et de concéder une viabilité à la nouvelle constitution sans aucun changement au niveau du régime. De surcroît, tous les problèmes qui ont été soulevés par les mouvements sociaux, et surtout par le M20F, trouvent réponse dans la nouvelle constitution. La solution au développement social et économique, la consolidation de l'État de droit et des droits de l'Homme, sont les facteurs d'une dynamique qui proviendra en fin de compte du Palais.

Malgré ce discours et tous les événements qui l'ont accompagné, le dialogue discursif se poursuit, et le M20F annonça un boycott du référendum à travers la campagne Mamsawetinch (nous ne votons pas). Cette compagne a été menée comme d'habitude par le moyen des vidéos sur Youtube, des réseaux sociaux et des communiqués de la plateforme Mamfakinch. Mais après un OUI voté par $98 \%$ des électeurs, le M20F commença à perdre du terrain. Une autre marche nationale était organisée le 11 septembre 2011 dont l'argument principal était

\footnotetext{
${ }^{11}$ Le 2 juin Kamal Ammari tombé victime des violences qu'il a subies par des forces de l'ordre lors d'une manifestation organisée par la coordination du M20F dans la ville d'Asfi.
} 
toujours la précarité des services publics et le coût élevé de la vie. Le M20F revient sur les mêmes arguments sans une nouvelle stratégie politique pour s'organiser et exercer plus de pression sur l'État. Le M20F a suivi cette dynamique de protestation en essayant de convoquer une marche; la dernière était le 22 janvier 2012.

La conclusion qu'on peut tirer à partir de l'analyse de cet ordre discursif, est qu'il existe deux espaces discursifs opposés, l'un dominant, avec une capacité de contrôle sur les masses ; il s'agit du discours du roi. Dans ce discours, la représentation de l'État, ses appareils et ses actions est faite d'une manière positive. L'autre espace discursif, dominé mais alternatif, touche des zones sensibles, à savoir, la représentation négative du pouvoir et de ses actions. Les deux représentations s'engagent dans une relation de conflit, de résistance et d'exercice de pression. Dans le point suivant, nous mettrons l'accent sur les stratégies discursives utilisées dans le discours du M20F pour légitimer sa position et sa représentation et pour délégitimer en même temps le pouvoir, le Makhzen et ses élites, son fonctionnement, ses actions et son appareil idéologique.

\section{Le Corpus d'analyse}

Avant de procéder à l'analyse de discours, nous allons d'abord présenter le champ d'action auquel appartiennent les discours du M20F. En ce qui concerne la notion de champ d'action, Wodak (2000, p. 132) la définit comme une série des sous-domaines de la réalité sociale qui jouent un rôle dans le processus d'établissement et de construction d'un cadre pour toute activité ou manifestation discursive ou communicative. L'auteur ajoute également que « la distinction espace-métaphorique entre les différents champs d'action peut être comprise comme celle qui existe entre les différentes fonctions sociales ou les objectifs sociaux institutionnalisées de certains pratiques discursives dans le domaine de l'action politique ». Ainsi, selon Wodak (2001, p. 124) le champ d'action du discours du M20F dans son ensemble est « la formation d'opinion, l'autoreprésentation et la représentation du politique ». De même, le discours du M20F étant issu d'un mouvement social de protestation et de contestation, il est aussi un discours de résistance dans un espace social, politique et idéologique où le conflit existe. De cette manière, nous considérons aussi que son champ d'action est la légitimation de soi et la délégitimation de l'autre, à savoir, le pouvoir et l'État.

En ce qui concerne le choix des discours, il faut signaler qu'il a été fait en partant de deux critères : le premier est l'espace discursif : nous avons choisi les exemples d'analyse en fonction de cette notion d'espace. Nous avons précisé précédemment que la production du discours du M20F s'exerce au niveau de deux espaces : la rue et les réseaux sociaux. C'est pour cela que les exemples que nous allons analyser font partie d'un corpus composé par: 8 communiqués publiés sur internet et sur les différents réseaux sociaux, 6 vidéos mises sur Youtube et une vingtaine de slogans répétés dans la rue durant les marches et les manifestations qui ont été organisées par le M20F jusqu'à maintenant.

Nous devons reconnaître également que nous avons rencontré des difficultés pour recueillir notre corpus, à cause de l'absence d'organisation au niveau de la 
diffusion et la circulation des documents qui concernent les représentations discursives, les opinions et les actions du M20F.

\section{Analyse linguistique-discursive}

\section{L'autoreprésentation comme stratégie discursive de légitimation du M20F}

Dans ce point nous allons analyser les formes de dénomination choisies par le M20F comme traits d'identité pour légitimer sa naissance et ses revendications. Le but principal est de pouvoir envisager ses représentations en termes de construction discursive de l'identité qui définit le M20F en tant que mouvement de contestation sociale et politique qui veut consolider sa légitimation sociopolitique face à l'État.

Exemple $\mathrm{n}^{\circ} 1$ :

$$
\text { "حنا شباب مغربي كانبغيو هاد البلاد ؤ كاناجدو التغيير والكرامة.12" }
$$

« Nous sommes des jeunes marocains qui aiment ce pays et qui revendiquent le changement et la dignité ".

Ce premier exemple définit clairement l'objectif de l'émergence du M20F, à savoir, le changement, d'un coté. De l'autre coté, on constate que l'action de " changer » est construite discursivement autour de deux éléments essentiels: le premier c'est l'âge, c'est-à-dire, que les militants qui composent ce mouvement sont des jeunes. Et le deuxième élément a un rapport avec deux valeurs. L'une est le patriotisme, l'amour du pays; et l'autre est de nature sociale ; la dignité.

Dans cette même ligne de représentation de soi-même, le M20F, s'est défini comme un mouvement indépendant et de changement, et c'est ce qu'on constate dans ce deuxième exemple.

Exemple $n^{\circ} 2$ :

"اللي خاص كلثي يعرفو ؤ يفهمو، حنا ما مور انا حتى شي تتظيم سياسي. حنا شباب مور انا هموم الثعب

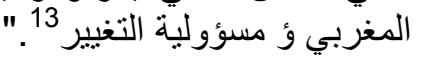

« Tout le monde doit savoir et comprendre qu'aucune organisation politique n'est derrière nous. Nous sommes des jeunes poussés par les inquiétudes du peuple marocain et par une responsabilité dans le changement $»$.

Cet exemple reflète l'idée que nous avons évoquée auparavant sur l'identité du M20F, un mouvement issu du peuple et pour le peuple. Avoir recours à deux arguments sociaux, "les inquiétudes du peuple marocain et la responsabilité dans le changement ", est une stratégie de légitimation sociale qui justifie l'émergence du M20F. Mais ce que nous devons aussi signaler c'est qu'il y a une sorte d'ambigüité au niveau de ce changement qui n'est pas bien défini dans la mesure où on le laisse ouvert à tous les domaines. Enfin, l'évocation de deux arguments sociaux uniquement n'est pas synonyme d'absence d'étiquette

\footnotetext{
${ }^{12}$ Vidéo deuxième campagne qui annonce la création du M20F et qui lance un appel à la manifestation du 20 février.

${ }^{13}$ Vidéo deuxième campagne qui annonce la création du M20F et qui lance un appel aussi à la manifestation du 20 février
} 
politique d'ailleurs clairement annoncée quand le M20F déclare qu'il est indépendant politiquement car aucune organisation n'est derrière sa naissance et ses revendications. Nous pouvons voir en l'absence d'un référent politique une stratégie de renforcement de cette légitimité sociale que voulait acquérir le M20F, sachant qu'il existe un manque de confiance de la part de la société envers le rôle des partis et des organisations politiques au Maroc.

Les deux exemples que nous allons présenter sont des extraits d'un communiqué publié suite à une marche nationale qui a eu lieu le premier jour du Ramadan, 4 mois après la naissance du M20F. Nous constaterons à travers ces exemples une évolution de la représentation et dans la construction discursive concernant l'identité du mouvement.

\section{Exemple $n^{\circ} 3$ :}

«Ce Mouvement qui soulève des revendications de Réformes profondes (changement de la forme et non la nature du régime) devient de plus en plus fort et guide des centaines de milliers de citoyens dans des manifestations hebdomadaires et parfois quotidiennes depuis le 20 Février 2011. [...] Ce mouvement qui a adopté officiellement le choix PACIFIQUE et NONVIOLENT et qui prend un élan considérable à la façon d'une boule de neige qui grandit avec le temps, agite les eaux stagnantes du paysage politique au Maroc, bouleverse la rigidité du système qui se voit en obligeance de métamorphoser sa forme et de procéder à un changement réel et rapide que le citoyen ordinaire touchera ses retombées concrètes! ${ }^{14} . »$

II semble bien qu'à mesure où le mouvement connaissait une évolution, ses représentations deviennent plus organisées, bien définis et de plus en plus critiques. On observe aussi que le mouvement a donné un sens à cette notion de "changement " (changement de la forme et non la nature du régime, la rigidité du système qui se voit en obligeance de métamorphoser sa forme et de procéder à un changement réel et rapide). En même temps, son message est nettement dirigé contre le pouvoir et son système. La manière avec laquelle le M20F construit cette représentation discursive socialement et ensuite politiquement, est liée non seulement à cette dynamique de changement qui constitue l'essence de son émergence, mais elle est liée aussi à la circulation d'un nouveau savoir qui contribue à la constitution d'un modèle social et politique alternatif.

II faut noter toutefois que l'une des formes de représentation choisie par le M20F pour la construction de son identité est la visibilité (en touchant des zones du Makhzen infranchissables jusqu'à présent). Les exemples suivants le confirment.

Exemple $n^{\circ} 4$ :

$$
\text { "ما بقيناش كانخافو من الزرواطة، ؤالإعتقالات السياسية ما كاتخلعناش بالعكس كا تعطينا قوة مصرار }
$$

« Nous n'avons pas peur des matraques et non plus des détentions politiques, au contraire ça nous donne de la force pour continuer et de la crédibilité ».

\footnotetext{
${ }^{14}$ Communiqué de presse du M20F après la marche du premier jour du Ramadan.

${ }^{15}$ Communiqué de presse du M20F après la marche du premier jour du Ramadan.
} 
Exemple $n^{\circ} 5$ :

« La conjoncture régionale et internationale caractérisée par le printemps Arabe et l'air révolutionnaire des indignés en Europe et dans le monde entier, influencent certainement la rue politique au Maroc et donne un appui moral considérable à ce mouvement de jeunes décidés qui manifeste audacieusement et qui ne cache pas ses intentions qui, à un temps tout proche, étaient parmi les tabous et les lignes rouges infranchissables! ${ }^{16}$.»

Le fait que le M20F évoque cette modification dans la relation avec l'État, conduit ce dernier à repenser la distribution des rapports de force qui existent dans le champ politique et social. Cela veut dire que le M20F prétend passer à l'action mais pas seulement pour changer cette relation historique d'obéissance vers l'autoritarisme de l'État, mais aussi pour exercer une influence discursive et postérieurement effective sur l'exercice de l'action politique et sociale. Cette visibilité est garantie de deux manières: premièrement, par le profil des manifestants dont la plupart sont des jeunes; deuxièmement, par le moyen de l'espace et la scène d'action que le M20F a choisis pour exercer son activisme : l'espace virtuel et la rue.

L'exemple suivant, $\mathrm{n}^{\circ} 6$, reflète cette idée d'action participative que le M20F revendique et exerce; un droit sociopolitique mais aussi une force et une ressource symboliques:

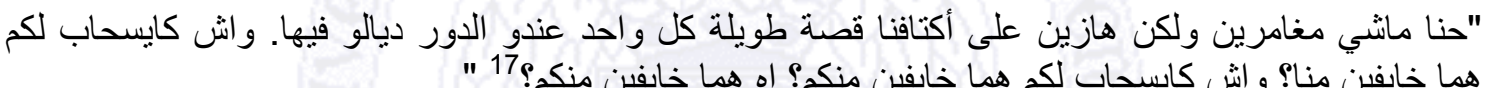

« Nous ne sommes pas des aventuriers, mais nous avons une longe histoire à raconter où chacun joue son propre rôle. Pensez-vous qu'ils ont peur de nous ? Pensez vous qu'ils ont peur de vous ? Oui, ils ont peur de nous $"$.

Nous assistons dans cet exemple à un recours à la métaphore «longue histoire ", dans le sens ici d'un long processus où nous devons participer tous. En outre, la négation du caractère aventurier des militants renvoie à l'idée de la réalité et non "la fiction » de ce processus que le M20F est entrain de commencer. De la même manière, quand le M20F utilise le mot "peur », et annonce son absence, il détruit les structures sociales et politiques, parfois symboliques, qui existent historiquement dans cette scène politique du Maroc et qui entravent n'importe quelles actions sur le politique issues du peuple ou d'un mouvement de contestation émergeant de la société civile. II faut indiquer aussi l'importance de la catégorie " nous » face à " eux » (ils), qui renvoie également à l'idée de l'inclusion et l'exclusion. L'inclusion de tous ceux qui veulent participer à cette histoire ou à ce processus (le « nous " évoque aussi, le prestige, la solidarité et la participation collective). Par contre, l'exclusion renvoie á ceux qui ont peur de ce processus. À travers donc la construction de cette dualité entre "nous » et "eux », le M20F met en évidence l'existence d'un conflit dont les deux protagonistes sont l'État, le pouvoir et son système, d'un coté, et les acteurs militants du M20F de l'autre coté.

L'exemple, $\mathrm{n}^{\circ} 7$, que nous analyserons évoque directement les acteurs qui ont peur de la naissance du M20F et des actions qu'il proclamait :

\footnotetext{
${ }^{16}$ Vidéo deuxième campagne qui annonce la création du M20F et qui lance un appel aussi à la manifestation du 20 février

${ }^{17}$ Vidéo mise sur Youtube qui fait appel à la manifestation du 22 janvier 2012.
} 
« Depuis la réussite de la marche populaire organisée le 6 août dernier, attirant les masses défavorisées de la ville de Rabat, la diversification des actions et formes d'expression que le mouvement proclame, font de plus en plus peur aux mafias du Makhzen politique et économique ${ }^{18} . »$

Ici on constate comment le M20F annonce cette diversité qui caractérise et va caractériser ses actions. Cette stratégie de diversification des actions du M20F rejoint ce que Hammami (2011, p.55) avait signalé au sujet de l'émergence d'un nouvel espace virtuel pour représenter cette scène révolutionnaire en Tunisie en disant qu'il s'agit " d'un processus lent de formation d'un espace public parallèle et alternatif, où sont apparues pluralité sociale et diversité politique ». Cette même description utilisée par le M20F au sujet de la diversification de ses actions, attribue une sorte de rationalisation au niveau des ses objectifs et des résultats qu'il cherche à atteindre (la réussite de la marche populaire attirant les masses défavorisées font de plus en plus peur). Cette description annonce de cette manière une vérité référentielle du « dit » sur soi-même et par lui-même.

Soutenue et vérifiée par une analyse linguistique, l'interprétation de cette représentation discursive effectuée par le M20F concernant ses traits d'identité permet de voir que l'action est fondamentale pour légitimer son existence en tant que mouvement de contestation sociale et politique. La construction discursive de ces actions qui établit la raison d'être du mouvement est faite en opposition aux actions menées par le Makhzen. De même, ces stratégies de représentation de soi-même est une garantie, symbolique et discursive, de cette légitimation sociale et politique que le M20F a essayé d'imposer dés le premier jour de sa naissance. Enfin, la dénomination des actions Makhzeniennes reste toujours négative, contrairement à ce caractère positif et nécessaire des actions du M20F.

\section{Les stratégies discursives de dénomination du pouvoir et de l'État: vers la délégitimation}

Dans le point suivant, nous analyserons les stratégies discursives qu'utilise le M20F dans la représentation du Makhzen afin de délégitimer les actions de ce dernier et procéder ensuite à la proposition et la légitimation de ses revendications.

Le premier exemple rappelle cette relation directe qui existe entre l'État et le pouvoir monarchique. Aussi, cette relation est-elle évoquée pour démontrer le fonctionnement de base de l'exercice de la politique du Maroc.

Exemple $n^{\circ} 8$ :

"إن النظام الملكي بملكه الجديد قد أعرب في بداية حكمه عن نيته في تحديث المجتمع المغربي و قام بمبادرات في

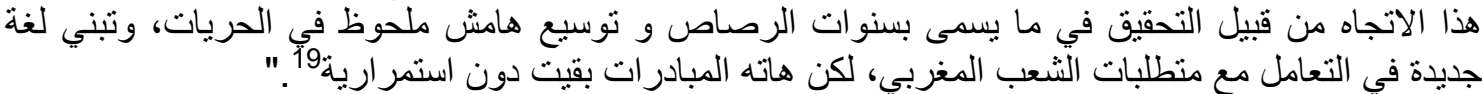

"Le régime monarchique à travers son nouveau roi a exprimé au début son intention de moderniser la société marocaine. Dans ce sens il a mis en place des initiatives dans le cadre de ces efforts d'enquête sur ce qu'on appelle les années de plomb. De même, il a élargi les marges de liberté de manière

\footnotetext{
${ }^{18}$ Communiqué de presse du M20F, coordination de Rabat, 13 août 2011.

${ }^{19}$ Le manifeste qui établit la création du Mouvement 20 Février au Maroc.
} 
significative, et a eu recours à un nouveau langage dans son traitement des exigences du peuple marocain. Mais ces initiatives sont restées sans continuité ".

On peut même ajouter que la description du régime et ses actions est construite d'une manière ascendante dans la mesure où on constate qu'au début il y a une représentation plus au moins positive du Makhzen. On confirme toutefois que la politique du Makhzen n'a pas donné de résultat. L'autre interprétation que nous pouvons faire à partir de cet exemple c'est l'introduction de la figure du roi en tant que représentant du régime marocain et en tant qu'agent discursif et politique fondamental dans la mise en place des actions. Nous assistons à une exposition claire de ce que représente le Makhzen et l'État.

Exemple $n^{0} 9$ :

"في ظل ما يعيشه الثـب المغربي اليوم من احتقان اجتماعي والإحساس بالإهانة والدونية، وتراجع القدرة

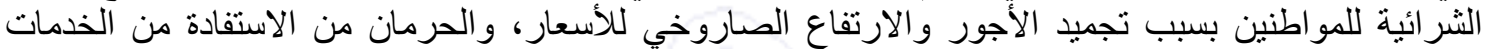

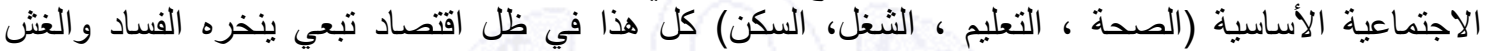

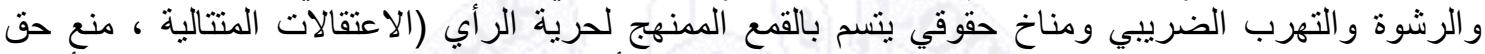

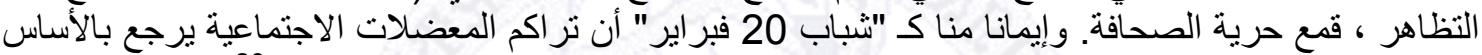

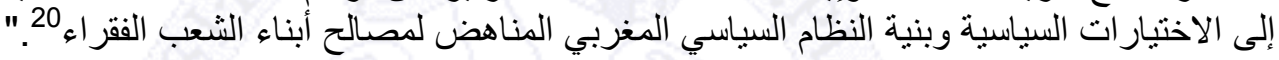

« À la lumière de ce que la population marocaine est en train de vivre aujourd'hui en terme de congestion et l'existence d'un sentiment d'humiliation sociale et d'infériorité, et la baisse du pouvoir d'achat des citoyens en raison du gel des salaires et l'accroissement spectaculaire des prix. En plus du non accès aux services sociaux de base (santé, éducation, emploi et logement). Tout cela dans une économie qui se caractérise par la dépendance, la corruption et la fraude fiscale. De plus, il faut mentionner le climat des droits de l'homme qui est caractérisée par la répression systématique de la liberté d'opinion (les arrestations successives, l'interdiction du droit de manifester, et la suppression de la liberté de la presse). Nous sommes donc convaincus nous les jeunes du 20 février que l'accumulation des problèmes sociaux est principalement due à des choix politiques et à la structure du régime politique marocain qui va contre les intérêts des populations pauvres ".

Cet exemple $\mathrm{n}^{\circ} 9$, par sa rhétorique que l'on pourrait qualifier de critique pessimiste dans le sens où le M20F à recours à une stratégie d'énumération d'un nombre de mauvaises situations que la population marocaine subit au niveau de tous les secteurs concernant le public (congestion et existence d'un sentiment d'humiliation sociale et d'infériorité, et la baisse du pouvoir d'achat des citoyens en raison du gel des salaires et l'accroissement spectaculaire des prix, non accès aux services sociaux de base (santé, éducation, emploi et logement). Le tout étant mis en relation avec cette politique d'actions économiques (caractérise par la dépendance, la corruption et la fraude fiscale) de l'État. Tout cela est accompagné d'un mauvais climat des droits de l'homme. Le recours à cette stratégie de description ascendante des différentes situations graves que subit le peuple culmine par une représentation totalement négative du choix politique adopté par le régime marocain. Nous assistons ici à une construction d'un argument pour délégitimer le Makhzen ; ce discours est basé

${ }^{20}$ Communiqué de presse du M20F qui lance un appel à la marche du 20 Février 2011 
sur une double représentation négative du Makhzen et de sa politique, ensemble de choix et d'actions qui ont totalement échoué.

Exemple $\mathrm{n}^{\circ}$ 10:

"هذا النظام الذي ماز ال يتمادى في تعنته ونهج سياسة الاذان الصماء والقمع و الاعتقالات و الاغنيالات من جهة.

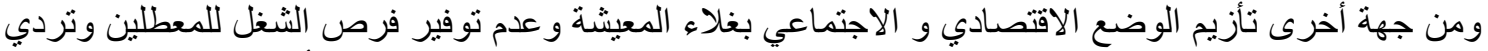

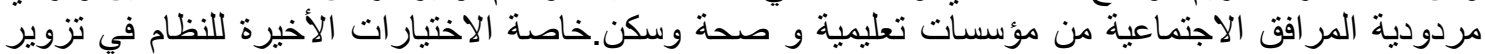

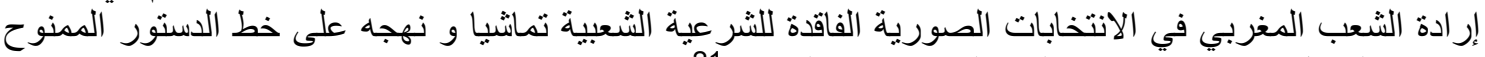

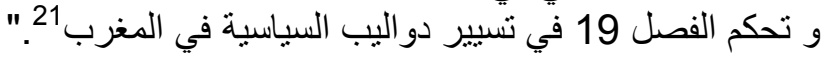

« Ce système se complaît toujours dans l'intransigeance et la politique de la sourde oreille et dans la répression, les arrestations et les assassinats. Sans oublier l'aggravation de la situation économique et sociale, le coût élevé de la vie et l'absence d'une garantie d'emploi ; le faible rendement des services sociaux, surtout les établissements d'enseignement, de la santé et du logement. Ceci est dû aux choix récents du système qui trahi la volonté du peuple marocain par les élections falsifiées sans légitimité aucune; à cause de cette Constitution octroyée et le rôle du Chapitre 19 concernant le processus d'orientation de la politique du Maroc ».

Enfin, l'exemple sert à démontrer la continuité du M20F dans cette dynamique de délégitimation du Makhzen par le biais d'une construction discursive d'autres actions menées par le système (l'intransigeance et la politique de la sourde oreille ; la répression, les arrestations et les assassinats). II faut noter toutefois que le M20F utilise un lexique qui remet en question et critique durement la question des droits et liberté, d'un côté. Et de l'autre côté, le discours évoque la constitution en tant que voix d'autorité démocratique en la qualifiant d' " octroyée ». Dans ce sens, c'est le nouveau projet de constitution en tant que nouvelle action proposée par l'État qui sera l'axe central de ce processus de représentation sociale et politique dans le discours du M20F. L'exemple suivant $\mathrm{n}^{\circ} 11$, démontre parfaitement comment le discours enlève toute crédibilité à ce projet :

« Le Makhzen présente la participation à ce référendum comme un « devoir citoyen et démocratique ». Pourtant, une multitude d'irrégularités lui ôtent, d'avance, toute crédibilité et toute légitimité. Par refus de cautionner un processus fondamentalement non démocratique. [...] 1 - Un processus antidémocratique et non transparent. 2 - Un débat constitutionnel bâclé. 3- Un manquement du Roi à son rôle d'arbitre. 4 - Un non respect des lois de la campagne pour le référendum. 5 - Une instrumentalisation irresponsable et dangereuse de la religion. 6 - Des actes d'agression et de harcèlement. 7 Une répartition du temps à l'antenne inégalitaire. 8 - Une atteinte dangereuse à la liberté d'expression. 9 - Des fraudes électorales à craindre. 10 - Violations flagrantes du Code électoral. 11 - Des actes de corruption des citoyens afin de les inciter à voter OUI. 12 - Des versements de sommes importantes à des partis politiques pour soutenir le OUI. 13 - Une ingérence étrangère inacceptable ${ }^{22} . »$

Cette description du projet de la constitution et de la constitution elle-même, fait état de l'existence de deux modèles de représentations sociales, politiques et

\footnotetext{
${ }^{21}$ Manifeste du M20F apparu après la marche du 11 janvier 2011.

${ }^{22}$ Communiqué Mamfakinch sur les atteintes à l'intégrité du scrutin référendaire. 28 juin 2011.
} 
idéologiques complètement opposés, à savoir, celui de l'État et celui du M20F, tel que nous l'avons évoqué dans la partie concernant l'ordre discursif. De plus, la construction discursive de ce projet de constitution dans le discours du M20F est basée sur une vision du contexte social, politique, économique totalement négative par rapport à la vision qui est représentée dans le discours dominant. Cette construction discursive de la constitution s'appuie sur un ensemble de croyances et de principes partagés dans un cadre démocratique que n'importe quelle constitution devrait respecter. Mais la nouvelle constitution que propose l'État ne le garantit pas, cet État étant anti-démocratique, ne respectant pas les lois des campagnes référendaires. Le fait que le discours du M20F décrit la constitution de cette manière est une stratégie sémantique dont le but est de concéder une sorte d'autorité et de crédibilité politiques et juridiques à sa voix, d'une part. Cela sert, d'autre part, à démontrer que le M20F a une opinion critique sur une question clé dans ce processus de changement auquel il voulait participer et dans lequel il tenait à s'inscrire en tant qu'un acteur responsable et engagé.

\section{Conclusion}

Le fait que le discours du M20F se construit autour de deux processus: l'autolégitimation sociale et politique et la délégitimation du Makhzen à tous les niveaux, ceci indique clairement que ce discours a besoin de stratégies discursives particulières. Nous avons bien vu comment ce discours dans ces représentations discursives articule une combinaison des aspects pragmatiques sémantiques avec d'autres politico- idéologiques pour atteindre les objectifs visés. Ces deux aspects sont étroitement liés, car la légitimité pragmatique, (les conditions contextuelles qui légitiment l'acte de parole. et les expressions linguistiques, à savoir, la forme sémantique de la légitimation) ne prendraient une valeur que quand l'énonciateur et l'auditeur partagent un savoir sur le contexte, sur les normes de l'acte de communication et sur les représentations et croyance sociales (Chilton, 2009). En ce qui concerne le savoir sur le contexte, nous avons vu que pour renforcer sa légitimité, le discours du M20F construit son modèle de représentation de soi et du makhzen à partir d'une série de croyances culturelles, historiques, sociales et politiques partagées par le peuple marocain dans des espaces discursifs publics et privés. Tout cela sert à démontrer qu'il y a une cohérence entre le niveau pragmatique de la légitimation, les stratégies discursives auxquelles le M20F recourt et les objectifs sociaux et politiques qu'il souhaite obtenir. En d'autres termes, dans ce discours du M20F, le contexte immédiat où l'acte de communication a eu lieu coïncide avec les stratégies de dénomination, les actions et les processus qui constituent l'axe central de la représentation discursive, soit du M20F ou du Makhzen. Tout cela culmine, comme nous l'avons déjà annoncé, en la création d'un autre modèle alternatif, discursif et contextuel, pour la politique au Maroc; un modèle alternatif qui délégitime la version officielle et lui enlève la crédibilité, l'autorité et la fiabilité sociale et politique.

Rappelons aussi que l'usage des langues dans ce processus d'autolégitimation joue un rôle important dans ce que nous avons appelé la diversification des actions du M20F. Dans ce sens, pour communiquer et diffuser son discours, le M20F a eu recours à différentes variétés linguistiques notamment, l'arabe 
marocain, l'amazigh, l'arabe classique et le français, et par fois l'anglais. Cette stratégie communicative et linguistique contribua à rendre le profil des locuteurs explicitement diversifié permettant ainsi une ouverture sur le plus large public possible. Nous pensons que cela s'inscrivait dans une démarche de consolidation et de renforcement de la légitimation sociale et politique du M20F.

Enfin, toutes nos interprétations, soutenues et vérifiées par une analyse linguistique, nous permettent d'avancer que tous les genres discursifs produits par le M20F rentrent dans le cadre d'un Discours Politique, car le M20F a rendu le débat politique plus accessible et ouvert en l'exerçant dans de nouveaux espaces, la rue et les réseaux sociaux, au-delà de la sphère étroite du pouvoir et ses élites.

\section{Bibliographie :}

AL'AZĀWİ, Wișāl, 2011 « A-thawarāt Al-'arabiya wa Istiḥqaqāt A-taghyīr (Les révolutions arabes et les avantages du changement)», Shu'un Al-Awsat, $n^{\circ} 139$, été, pp.32-44

ALMANJDRA, Mehdi, 2003, Humiliation à l'ère du méga-impérialisme, Al Jadida, Annajah.

BECHIR AYARI, Michaël et GEISSER, Vincent, 2011, Rennaissances árabes. 7 clés sur des révolutions en marche. Paris, Lés Éditions de l'Atelier/Éditions ouvrières.

BEN NEFISSA, Sarah, 2011, " Mobilisations et révolutions dans les pays de la Méditerranée arabe à l'heure de "l'hybridation » du politique », Revue Tiers Monde, Protestations sociales, révolutions civiles. Transformation du politique dans la Méditerranée arabe. Hors série, Sarah Ben Néfissa et Blandine Destremau, (dir.), Paris, Armand Colin, pp. 5-24.

2011, « les angles morts de l'analyse politique des sociétés de la région ", Confluences méditerranée, $\mathrm{n}^{\circ} 77$, été, pp. 75-100.

BENNFLA, Karine, 2011, «Introduction », Confluences méditerranée, n 78, été, pp. 9-24.

BOUKOUS, Ahmed, 2008, "Le champ langagier: Diversité et stratification », Revue Asinag no 1, pp. 15-37.

BOURDIEU, Pierre, 1982, Ce que parler veut dire, Paris, Gallimard.

CATUSSE, Miriam, 2011, « Le " social » : une affaire d'Etat dans le Maroc de Mohammed VI », Confluences méditerranée, nº 78, été, pp. 63-76. 
CATUSSE, Miriam et VAIREL, Frédéric, 2010, «Question sociale et développement économique: les territoires de l'action publique et de la contestation au Maroc », Politique Africaine, $n^{\circ} 120$, pp. 5-23.

CATUSSE Miriam et VAIREL Frédéric, 2010, «Question sociale et développement: les territoires de l'action publique et de la contestation au Maroc », Politique africaine, $\mathrm{n}^{\circ} 120$, pp. 5-23.

CHILTON Paul, 2004, Analysing Poltical Discourse: Theory and Practice, London, Routledge.

CICOUREI, Aaron, 1980, «Three Models of Discourse Analysis : The Role of Social Structure », Discourse Processes, n² 3, pp. 101-132.

FERRIÉ, Jean Noël et DUPRET, Baudouin, 2011, « La nouvelle architecture constitutionnelle et les trois désamorçages de la vie politique marocaine ", Confluences méditerranée, $\mathrm{n}^{\circ} 78$, été, pp. 25-34.

FOUCAULT Micheal, 1971, L'ordre du discours, Paris, Gallimard.

GONZALEZ RIERA, José María, 2011, " Des années de plomb au 20 février: le rôle des organisations des droits de l'homme dans la transition politique au Maroc », Confluences méditerranée, n 78, été, pp. 35-47.

FAOUAD, Wissam, 2010, «Facebook y la juventud árabe. ¿Activismo social o liberación social », Awraq, nº 9, pp. 93-100.

HAMMAMI, Sadok, 2011, « Quand le peuple rentre en scène », Medium, n 29, pp.49-65.

HELLER, Heller, 2007, " Distributed Knowledge, distributed power: A Sociolinguistics of structuration », Text \& Talk, $n^{\circ} 27,5 / 6, p p .622-653$.

HIBOU, Béatrice, 2011, "Le mouvement du 20 février, le Makhzen et l'antipolitique. L'impensé des réformes au Maroc », mai 2011, http://www.cerisciences-po.org.

KELLER, Reiner, 2007, « L'analyse du discours du point du vue de la sociologie de la connaissance. Une perspective nouvelle pour les méthodes qualitatives », Recherches qualitatives, $n^{\circ} 3$, pp. 287-306.

MOUSTAOUI, Adil, (sous presse), "Language policy, identity and Dynamics of Change in Morocco: Toward a New Model », in Actes de Colloque Language and Cultures in Contact, Université Chouaib Doukali, Al Jadida.

SIDI HIDA, Bouchra, 2011, "Mobilisations collectives à l'épreuve des changements au Maroc », Tiers Monde, Protestations sociales, révolutions civiles. Transformation du politique dans la Méditerranée arabe. Hors série, Sarah Ben Néfissa et Blandine Destremau, (dir.), Paris, Armand Colin, p163188.

VAN DIJK Teun et MARTIN ROJO Luisa, 1997, " There was a Problem, and it was Solved! Legitimating the Expulsion of " Illegal » Migrants in Spanish Parliamentary Discourse », Discourse \& Society, n 8(4), p. 523-66.

VAN DIJK, Teun, 1999, Ideología. Una aproximación multidisciplinar. Barcelona, Gedisa. 
_, 2003, " La multidisciplinariedad del análisis crítico del discurso: un alegato a favor de la diversidad ». in Wodak Ruth et Meyer Micheal (dir.), Métodos del análisis crítico del discurso. Barcelona: Gedisa, 2003, pp. 143-177. 159-17

2006, « Discourse, context and cognition », Discourse Studies, vol 8 (1), pp.

VAN LEEUWEN, Teun, 1996, "The representation of social actors", in En Caldas-Coulthard. Carmen et Coulthard Micheal (dir.), Text and practice. London, Routledge, 1996, pp. 33-70.

Wodak, Ruth et al, 1999, "The discursive construction of national identity", Discourse \& Society, Vol 10 (2), pp. 149-173.

_, 2000, « ¿La sociolingüística necesita una teoría social? Nuevas perspectivas en el Análisis Crítico del Discurso », Discurso y Sociedad, vol. 2 (3), pp. $123-147$.

WODAK Ruth et REISIGL Michael, 2001, Discourse and discrimination. Rhetoric of Racism and Antisemitism, London / New York, Routledge.

WODAK Ruth et MEYER Michael (dir.), 2003, Métodos del análisis crítico del discurso, Barcelona, Gedisa.

WODAK Ruth et CHILTON Paul (dir.), 2005, A New Agenda in Critical Discourse Analysis. Amsterdam /Philadelphia, John Benjamins Publishing Company.

WODAK, Ruth, 2011, "Challenges to Critical Discourse Studies-Opening Borders and Transcending Dichotomies », Conférence présentée dans le cadre du CDA Workshop, 24 de mars 2011, Madrid. 